\title{
Narrow Luminescence Linewidth of a Silicon Quantum Dot
}

\author{
Ilya Sychugov, ${ }^{1}$ Robert Juhasz, ${ }^{1}$ Jan Valenta, ${ }^{1,2}$ and Jan Linnros ${ }^{1}$ \\ ${ }^{1}$ Laboratory of Materials and Semiconductor Physics, Royal Institute of Technology, SE-16440, Kista-Stockholm, Sweden \\ ${ }^{2}$ Faculty of Mathematics and Physics, Department of Chemical Physics and Optics, Charles University, \\ Ke Karlovu 3, Prague 2, CZ-12116, Czech Republic
}

(Received 25 August 2004; published 4 March 2005)

\begin{abstract}
Single-dot luminescence spectroscopy was used to study the emission linewidth of individual silicon nanocrystals from low temperatures up to room temperature. The results show a continuous line narrowing towards lower temperatures with a linewidth as sharp as $2 \mathrm{meV}$ at $35 \mathrm{~K}$. This value, clearly below the thermal broadening at this temperature, proves the atomiclike emission from silicon quantum dots subject to quantum confinement. The low temperature measurements further reveal a $\sim 6 \mathrm{meV}$ replica, whose origin is discussed. In addition, an $\sim 60 \mathrm{meV}$ TO-phonon replica was detected, which is only present in a fraction of the dots.
\end{abstract}

PACS numbers: 78.67.Hc, 78.55.Ap

Ideal semiconductor quantum dots have specific discrete, atomiclike energy levels yielding sharp emission lines for optical transitions between excited and ground states. As a consequence, the emission linewidth may be much smaller than the thermal energy $k_{B} T$. This has, indeed, been observed for nanocrystals of direct band gap semiconductor materials of type II-VI [1] and III-V, usually grown epitaxially by self-assembling techniques. The homogeneous linewidth of a quantum dot is one of its most important parameters where the temperature dependence reflects unique dephasing mechanisms of quantum states by phonons [2]. The sharp spectral features of individual quantum dots are, however, normally smeared because of inhomogeneous line broadening resulting from measurements on large ensembles of quantum dots. Here, individual size variations give rise to large variations of emission energies as a direct consequence of quantum confinement. Recent developments in microspectroscopy techniques have, however, enabled studies of individual quantum dots yielding ultranarrow emission linewidths [1].

The luminescent properties of silicon nanocrystals were first explored in porous $\mathrm{Si}$ [3] and were later studied in $\mathrm{Si}$ nanocrystals formed by various techniques, mostly resulting in nanocrystals passivated by silicon dioxide [4]. The observed spectra were usually very broad, a few hundred $\mathrm{meV}$ even at low temperatures, which was generally attributed to the inhomogeneous line broadening. Narrower photoluminescence (PL) bands were demonstrated using size selection methods subsequent to the fabrication step [5]. More detailed information could, however, be achieved using selective spectroscopy where resonant excitation deep in the PL band was employed [6] or by spectral hole burning [7]. This revealed relatively narrow emission lines and both no-phonon and phonon-assisted transitions. Still, an ensemble of quantum dots was addressed rather than a single nanocrystal, screening valuable information on its level structure, emission band, and possible individual variations from dot to dot.
In our previous work [8] we successfully used single-dot spectroscopy to obtain PL spectra of individual nanocrystals at room temperature. The main difficulties were the low emission rate and adequate separation of the nanocrystals in order to use far-field luminescence microscopy. The nanocrystals were formed by electron beam lithography, plasma etching, and subsequent size reduction by a careful oxidation scheme. These studies showed a singledot linewidth of $\sim 120-150 \mathrm{meV}$ with peak photon energy varying from dot to dot. In addition, blinking (PL intermittence between two discrete states, on and off) was observed [9], as well as a high degree of individual polarization demonstrating the individual nature of the luminescence properties of each nanocrystal. The relatively large linewidth at room temperature found even for an individual nanocrystal, however, raised some doubts on the emission mechanism being that of a pure Si quantum dot, although theoretical calculations [10] provided support for a broad spectrum even at $0 \mathrm{~K}$ because of phonon involvement.

In this Letter we show results from low temperature measurements demonstrating linewidths of individual nanocrystals as sharp as $2 \mathrm{meV}$ at $35 \mathrm{~K}$. This is clearly below $k_{B} T$ at this temperature demonstrating true quantum dot PL emission characteristics. Furthermore, we observe a $\sim 6 \mathrm{meV}$ replica of the main peak at low temperatures as well as a $\sim 60 \mathrm{meV}$ replica, attributed to transverse optical (TO) phonons. Intriguingly, this TO replica is only present in a fraction of the analyzed dots. We discuss these new findings in terms of different physical scenarios.

Samples in the form of pillar arrays were fabricated (see $[8,11]$ for details) using electron beam lithography and reactive ion etching followed by a two stage oxidation scheme to form nanocrystals at the pillar tops. $N$-type $\mathrm{Si}$ wafers with a $25 \mathrm{~nm}$ thermal oxide were used as substrates, and holes with a diameter of $\sim 100 \mathrm{~nm}$ were defined by the electron beam in a positive type of resist (ZEP520A). This was followed by liftoff of $\mathrm{NiCr}$ and reactive ion etching to produce $\sim 200 \mathrm{~nm}$ tall $\mathrm{Si}$ pillars. Thermal oxidation at 
$900{ }^{\circ} \mathrm{C}$ induced a shrinking of the Si cores of the pillars leading to the formation of a silicon nanocrystal at the top of the pillars as a result of self-limiting oxidation [12], known to occur for a small radius of curvature. In Figs. 1(a) and 1(b), scanning electron microscopy (SEM) images of the pillars are presented, taken after first oxidation and removal of the oxide by HF acid. Indeed, $<50 \mathrm{~nm}$ cores seem to have formed at the top of the pillars and, presumably, a second oxidation step leads to complete consumption of the $\mathrm{Si}$ in the trunk just below the top, while at the top of the pillars single silicon nanocrystals may still remain. This is further supported by SEM images (not shown), taken after a second oxidation and a subsequent removal of the oxide, revealing only sharp tips of $\mathrm{Si}$ cores most likely as a result of a simple "falloff" of separated nanocrystals from pillar tops during $\mathrm{SiO}_{2}$ etching.

For PL characterization the samples were mounted on the cold finger of a liquid helium flow cryostat. PL was excited by the UV line $(325 \mathrm{~nm})$ of a cw He-Cd laser with pumping intensity $\sim 1 \mathrm{~W} / \mathrm{cm}^{2}$. The light emitted from a sample was collected by a window-corrected objective at high numerical aperture $(\mathrm{NA}=0.65)$. An imaging spectrometer coupled to a liquid nitrogen cooled charge coupled device camera was employed to detect the luminescence. Figure 1(c) shows a white light reflected image of the pillar arrays while Fig. 1(d) shows the corresponding PL image under $325 \mathrm{~nm}$ excitation, revealing several well

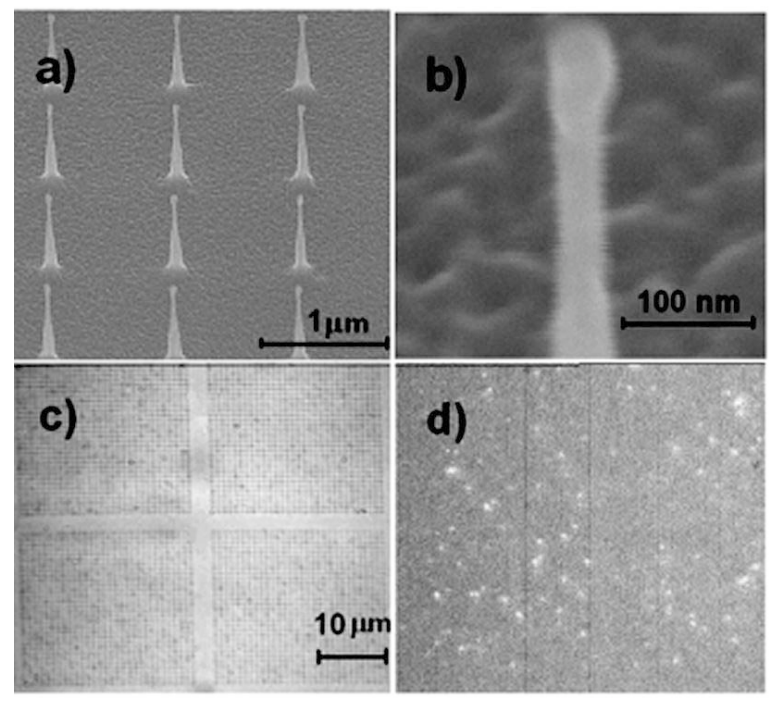

FIG. 1. (a) SEM images (tilt view) of Si nanopillars after first oxidation step. $\mathrm{SiO}_{2}$ was removed by $\mathrm{HF}$ etching in order to see remaining Si core. (b) Enlarged image of a pillar. Second oxidation is supposed to consume the "neck" between the nanocrystal at the top of the pillar and its basement, thus creating an isolated $\mathrm{Si}$ quantum dot embedded in a $\mathrm{SiO}_{2}$ matrix. (c) Optical microscope image of the white light reflection on pillar arrays (top view). Distance between neighboring pillars is $1 \mu \mathrm{m}$. (d) Corresponding PL image under UV-light excitation. Each bright spot can be traced back to a certain pillar. separated emission objects. Two different gratings provided spectral resolutions of 3 or $0.8 \mathrm{~nm}$, respectively. The laser beam impinged on the sample surface at grazing incidence. To get an acceptable signal to noise ratio each spectrum acquisition lasted at least $30 \mathrm{~min}$. All spectra were corrected with the system response curve.

Several indications of the observation of single Si quantum dot emitters have been found [cf. Fig. 1(d)] and we start by outlining a few features in support of this. First, observed photon emission energies from different nanocrystals lie in the range from 1.5 to $2.0 \mathrm{eV}(620-825 \mathrm{~nm})$; see Fig. 2. According to quantum-confinement theory and size-separation experiments (e.g., [13] and references therein) this range corresponds to $\mathrm{Si}$ nanocrystals, embedded into a $\mathrm{SiO}_{2}$ matrix, with sizes from 3 to $6 \mathrm{~nm}$ in diameter. For comparison, in Fig. 2 we have also included a typical emission spectrum from an ensemble of Si nanocrystals embedded in oxide (prepared by ion implantation and annealing [4]). Second, blinking (random switching between two intensity levels) was observed for most individual nanocrystals [9]. This phenomenon is known to be a hallmark of single fluorescent semiconductor nanocrystals as well as of single molecules $[1,14]$. Finally, we analyzed the temperature dependence of the PL emission from a single nanocrystal and found a band gap narrowing effect, just as for bulk silicon (also observed for direct band gap materials quantum dots, e.g., [15]), proving that the PL emission emanates from silicon entities.

Upon decreasing temperature we observed a continuous line narrowing of the luminescence band and the occurrence of certain sidebands in some cases. At temperatures below $30 \mathrm{~K}$, however, we were unable to detect any consistent PL. We attribute this to the drastic lifetime increase observed for Si nanocrystals and for porous Si at cryogenic

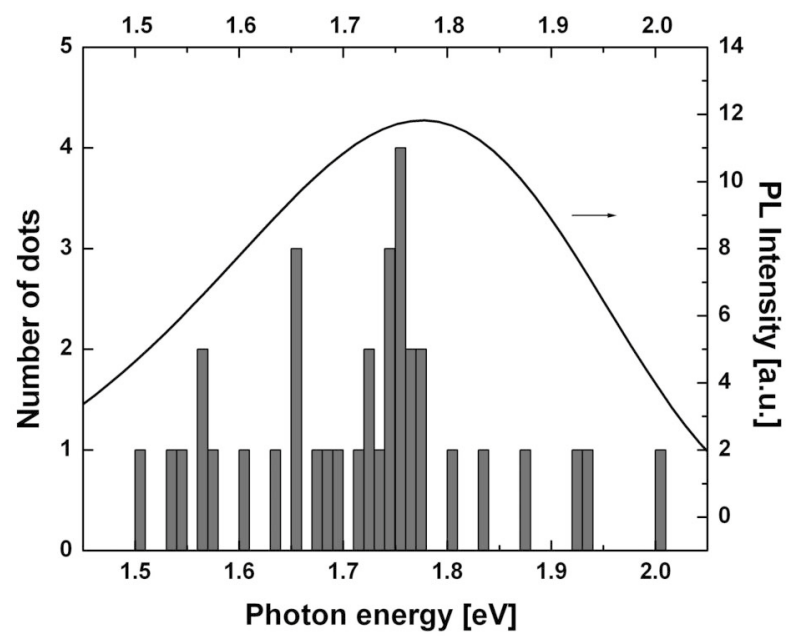

FIG. 2. Statistical breakdown of the number of dots with different emission photon energies (step $0.1 \mathrm{eV}$ ) measured at $80 \mathrm{~K}$. Solid line shows a typical ensemble PL spectrum from nanocrystals embedded in silicon dioxide for comparison (from [4]). 
temperatures [3]. This effect was explained in terms of a singlet-triplet splitting with a lower lying "dark" triplet state. As the emission rate is lowered by a factor of about 50 , it seems to be below the detection capabilities of our measurement system. Above $30 \mathrm{~K}$, on the other hand, we were able to record single-dot emission spectra, and in Figs. 3(a) and 3(b) PL spectra at $35 \mathrm{~K}$ of two different dots are presented. It is seen that the emission line consists of a sharp peak and a somewhat broader satellite, separated by $\sim 6 \mathrm{meV}$. This line might be attributed to a low frequency acoustic phonon-assisted transition, as discussed below.

The data on emission linewidth obtained for dots with well resolved $\sim 6 \mathrm{meV}$ replica are summarized in Fig. 4(a). Clearly, some of the nanocrystals exhibit sharp peaks with FWHM less than $k_{B} T$ proving the quantized nature of states in a Si quantum dot. It is interesting to note that no correlation of the emission linewidth with dot size was observed. In general, the homogeneous width of a quantum dot is given by the inverse of the dephasing time, which consists of the radiative lifetime and various scattering times (interaction of the exciton with phonons, interface states, defects, etc.) [2]. Thus, the linewidth is a unique parameter of an individual quantum dot and depends on the interface with surrounding matrix, dot geometry, and purity.

The observed temperature evolution of the emission line for different nanocrystals can be summarized as follows. For some nanocrystals two peaks separated by $\sim 6 \mathrm{meV}$ can be discerned at low temperatures [Figs. 3(a) and 3(b)]. With temperature increasing these two peaks are gradually broadening and eventually merge together [Fig. 3(d)]. In addition, some fraction of the nanocrystals revealed a second peak standing $\sim 60 \mathrm{meV}$ apart from the main one

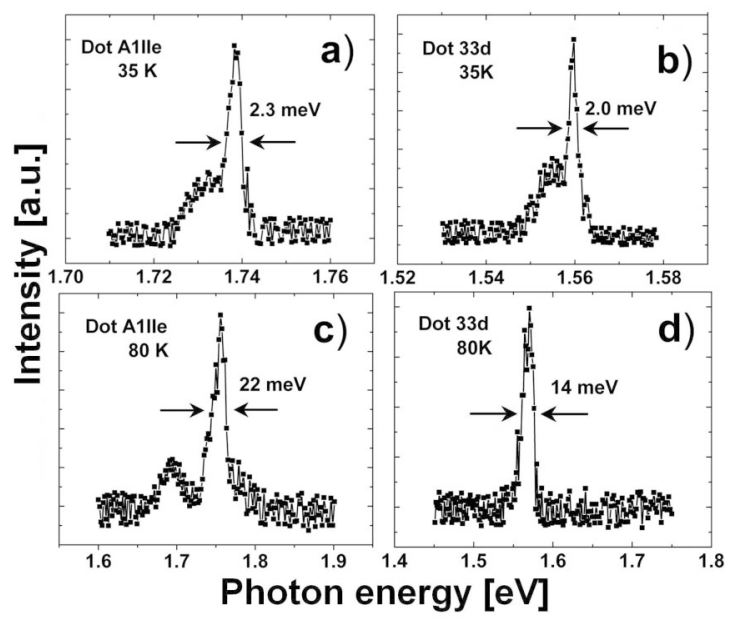

FIG. 3. PL spectra of two different single Si nanocrystals at (a),(b) $35 \mathrm{~K}$ and at (c),(d) $80 \mathrm{~K}$. FWHM of Lorentzian fits are shown. Extracted linewidth values are deconvoluted with the system resolution.
[Fig. 3(c)]. This group of quantum dots exhibits a quite broad emission line $(\sim 150 \mathrm{meV})$ at room temperature (RT). At the same time, the rest of the investigated nanocrystals exhibited only a gradual broadening of the main emission line with no other lines appearing all the way to RT. At RT the linewidth of these dots is somewhat narrower $(\sim 100 \mathrm{meV})$ than those with a $\sim 60 \mathrm{meV}$ sideband.

In discussing our findings we first note that the observed $\sim 60 \mathrm{meV}$ replica is close to the TO phonon energy for bulk $\mathrm{Si}$ (56 meV at the $\mathrm{X}$ point, $64 \mathrm{meV}$ at the $\Gamma$ point) and does not vary with peak emission energy (dot size). While this TO phonon involvement seems quite natural, we would like to point out that only a fraction $\sim 1 / 3$ of the dots exhibited this peak at $80 \mathrm{~K}$ and this fraction is slowly increasing with temperature. We can suggest that in some nanocrystals carriers are strongly localized (promoting nophonon PL) or that local differences in geometry may enhance or decrease the probability of the TO phononassisted process with temperature. Such a zero-phonon optical transition was reported earlier for Si nanocrystals

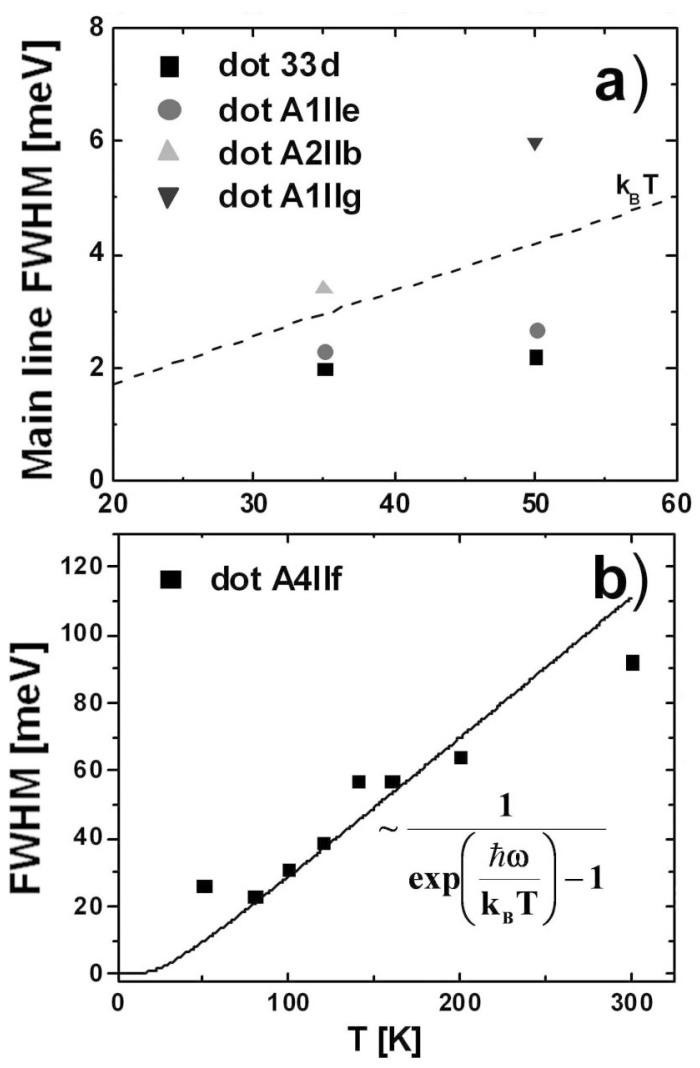

FIG. 4. (a) Lorentzian FWHM values of the main emission line for different dots at temperatures below $50 \mathrm{~K}$. Dashed line shows the energy equivalent of temperature in this range. Only dots with a resolvable acoustic phonon peak are shown. (b) Temperature dependence of the exciton linewidth for a dot without a TO-phonon replica (acoustic phonon line is not resolved at $50 \mathrm{~K}$ for this dot). The solid line is a fit based solely on Bose statistics for a low frequency acoustic mode ( $\hbar \omega=6 \mathrm{meV})$. 
[6] and predicted by theory [16] as a breakdown of $k$ conservation at small dimensions. The ratio of no-phonon transitions to the TO phonon-assisted ones would then increase with emission energy (reduced size). For our data we cannot find such a trend and, instead, we found a dot-to-dot variation of the phonon-assisted process, suggesting local differences, which are averaged in ensemble measurements. It is important to note, though, that Kovalev et al. [6] reported a large change of luminescence line ratio in favor of the no-phonon line for "heavily" oxidized samples, suggesting a strong dependence on the nanocrystal local environment. Moreover, theoretical calculations [10] made within the tight-binding model showed that the existence of strained interface regions in the oxidized nanocrystals, which is the case for the present experiment, leads to the localization of carriers and a drastic increase of the no-phonon line transition probability.

For the explanation of the $\sim 6 \mathrm{meV}$ replica, observed at low temperatures, one may invoke torsional or spheroidal modes as calculated by Takagahara [17]. For a $4 \mathrm{~nm}$ diameter Si nanocrystal he calculated a set of discrete values for the acoustic phonon energy spectrum starting from $\sim 5 \mathrm{meV}$. By Raman spectroscopy [18] the presence of confined acoustic modes with energy of a few meV was, indeed, experimentally observed. Since the range of nanocrystal sizes probed in the present work is quite limited, no distinct size dependence of the mode energy was revealed. In Fig. 4(b) the Lorentzian FWHM of the emission line is plotted versus temperature for a Si nanocrystal without a TO-phonon replica. It can be seen that a fit based solely on Bose statistics for a low frequency acoustic mode $(\hbar \omega=$ $6 \mathrm{meV}$ ) with the only fitting parameter - the proportionality coefficient-reproduces correctly the main trend in the linewidth temperature dependence. Although not a definite proof of this model, we conclude that exciton interaction with low frequency acoustic phonons is consistent with the observed linewidth temperature evolution. Possible biexciton nature of this peak is ruled out by the lack of excitation intensity influence on the peak intensity ratio. Similarly, it can hardly be the triplet state line since no emission was detected at lower temperatures, where this is the only possible recombination path.

In conclusion, single-dot photoluminescence spectroscopy has been successfully applied to investigate emission properties of $\mathrm{Si}$ quantum dots down to $35 \mathrm{~K}$. For the first time it was shown that the emission linewidth of Si nanocrystals can be, at least, as sharp as for direct band gap material quantum dots, confirming the atomiclike nature of these low-dimensional entities. Also, exciton-phonon interactions in Si nanocrystals were revealed as a TO-phonon replica was observed and a $\sim 6 \mathrm{meV}$ replica which we tentatively attribute to spheroidal or torsion acoustic modes. This latter mode appears to be responsible for the temperature dependence of the observed linewidth leading to the broad PL spectrum from a single silicon nanocrystal at room temperature, as reported earlier. Finally, this work demonstrates the importance of single-dot studies as PL emission mechanisms may vary from dot to dot due to local variations determined, e.g., by geometry or strain, inaccessible by ensemble studies.

The authors gratefully thank A. Galeckas for technical assistance and D. Haviland of the KTH Nanofabrication Laboratory for the access to the $e$-beam lithography setup. We also thank C. Delerue for fruitful discussions. Financial support was received from the Swedish Research Council (V.R.). One of the authors (J. V.) acknowledges funding from the GACR (Grant No. 202/03/0789) and GAAV (Grant No. IAA1010316).

[1] S. A. Empedocles, D. J. Norris, and M. G. Bawendi, Phys. Rev. Lett. 77, 3873 (1996).

[2] T. Takagahara, Phys. Rev. B 60, 2638 (1999); Phys. Rev. Lett. 71, 3577 (1993).

[3] A. G. Cullis, L. T. Canham, and P. D. J. Calcott, J. Appl. Phys., 82, 909 (1997).

[4] J. Linnros et al., J. Appl. Phys. 86, 6128 (1999).

[5] W. L. Wilson, P. F. Szajowski, and L. E. Brus, Science 262, 1242 (1993).

[6] D. Kovalev et al., Phys. Rev. Lett. 81, 2803 (1998).

[7] D. Kovalev et al., Phys. Rev. B 57, 3741 (1998).

[8] J. Valenta, R. Juhasz, and J. Linnros, Appl. Phys. Lett. 80, 1070 (2002).

[9] I. Sychugov, R. Juhasz, J. Linnros, and J. Valenta, Phys. Rev. B (to be published).

[10] C. Delerue, G. Allan, and M. Lannoo, Phys. Rev. B 64, 193402 (2001).

[11] R. Juhasz and J. Linnros, Appl. Phys. Lett. 78, 3118 (2001).

[12] H. I. Liu et al., Appl. Phys. Lett. 64, 1383 (1994).

[13] G. Ledoux et al., Appl. Phys. Lett. 80, 4834 (2002).

[14] W. E. Moerner, Science 277, 1059 (1997).

[15] I. Favero et al., Phys. Rev. B 68, 233301 (2003).

[16] M.S. Hybertsen, Phys. Rev. Lett. 72, 1514 (1994).

[17] T. Takagahara, J. Lumin. 70, 129 (1996).

[18] M. Fujii, Y. Kanzawa, S. Hayashi, and K. Yamamoto, Phys. Rev. B 54, R8373 (1996). 